4] R. Fortet and E. Mourier, Les fonctions aléatoires comme eléments aléatoires dans les espaces Banach, Studia Math. 15 (1955), pp. 62-79.

[5] J. Hoffman-Jørgenson, The strong law of large numbers and central limit theorem in Banach spaces, Aarhus Universitet Matematisk Institut, September 1974 .

[6] J. Kuelbs, A strong convergenee theorem for Banach space valued random variables, preprint.

[7] - Positive definite symmetric functions on linear spaces, J. Math. Anal. Appl. 42,2 (1973), pp. 413-426.

[8] Yu. V. Prohorot, The method of characteristic functionals, Fourth Berkeley Symposium 2 (1961), pp. 403-419.

[9] N. N. Vakhania, Sur les répartitions de probabilités dans les espaces de suites numériques, C. R. Acad. Sci. Paris 260 (1965), pp. 1560-1562.

[10] M. J. Wiohura, A note on the convergence of series of stochastic processes, Annals of Prob. 1.1 (1973), pp. 182-184.

[11] W. A. Woyczyński, Random series and laws of large numbers in some Banach spaces, Theory of Prob. and Applic. 18 (1973), pp. 350-355.

DEPARTMENT OF MATHEMATICS

UNIVERSITY OF WISCONTIN, MADISON, WISC.

Received May 26, 1975

\section{Interpolation of Orlicz spaces}

by

JAN GUSTAVSSON and JAAK PEETRE (Lund)

Abstract. Let $\varphi, \varphi_{0}$ and $\varphi_{1}$ be positive increasing functions on $[0, \infty)$ connected by the formula $\varphi^{-1}=\varphi_{0}^{-1} \varrho\left(\varphi_{1}^{-1} / \varphi_{0}^{-1}\right)$ with a suitable $\varrho$. Consider the corresponding Orlicz spaces $L^{\varphi}, L^{\varphi_{0}}$ and $L^{\varphi_{1}}$. It is shown that $L^{\varphi}$ is an interpolation space with respect to $L^{\varphi_{0}}$ and $L^{\varphi_{1}}$ provided $\varrho$ is "a little more than concave".

0. Introduction. In this paper we give a contribution to the following problem: Given three Orlicz spaces $L^{\varphi}, L^{\varphi_{0}}$ and $L^{\varphi_{1}}$ on some measure space $M$, under what conditions is it true that $L^{\varphi}$ is an interpolation space with respect to $L^{\varphi_{0}}$ and $L^{\varphi_{1}}$ ? Roughly speaking, we show that, assuming that $\varphi$ is expressed in terms of $\varphi_{0}$ and $\varphi_{1}$ in the form

$$
\varphi^{-1}=\varphi_{0}^{-1} \varrho\left(\varphi_{1}^{-1} / \varphi_{0}^{-1}\right)
$$

(where $\varphi^{-1}$ is the inverse of $\varphi$, etc.), it is sufficient to assume that $\varrho$ is "a litile more than concave". In particular, our result applies when

$$
\varrho(x)=x^{\theta} \quad(0<\theta<1),
$$

in which case $(0.1)$ specializes to

$$
\varphi^{-1}=\left(\varphi_{0}^{-1}\right)^{1-\theta}\left(\varphi_{1}^{-1}\right)^{\theta},
$$

covering thus the case treated by Rao [17] (cf. Kraynek [10]). As an example of a function $\varrho$, more general than the one in $(0.2)$, to which our theory applies, we mention

$$
\varrho(x)=x^{\theta}(\log (e+x))^{\alpha}(\log (e+1 / x))^{\beta}
$$

$$
(0<\theta<1, \alpha, \beta \text { arbitrary real). }
$$

Whereas that author uses Thorin's proof conveniently adapted, we shall instead rely on an idea of Gagliardo [5], in the special case of $L^{p}$ (cf. Peetre [13]). More precisely, given any quasi-Banach couple $\vec{A}=\left\{A_{0}, A_{1}\right\}$ we define interpolation spaces $\langle\vec{A}, \varrho\rangle=\left\langle A_{0}, A_{1}, \varrho\right\rangle$. In the special case when $A_{0}$ and $A_{1}$ are both rearrangement invariant spaces of measurable 
functions on our measure space $M$ we also define spaces $A_{0} \varrho\left(A_{1} / A_{0}\right)$ which generalize the spaces $A_{0}^{1-\theta} A_{1}^{\theta}$ introduced by Calderón [4]. Now our full result reads (cf. Theorem 7.3 )

$$
L^{\varphi}=\left\langle L^{\varphi_{0}}, L^{\varphi_{1}}, \varrho\right\rangle=L^{\varphi_{0}} \varrho\left(L^{\varphi_{1}} / L^{\varphi_{0}}\right) .
$$

The restriction on $\varrho$ seems to be of a merely technical nature. In fact, we strongly suspect that the right necessary and sufficient condition on $o$ is pseudoconcave, i.e. concave up to equivalence.

It may be of some interest to notie that the corresponding problem for Orlicz classes has been essentially solved (see Peetre [12]). (For convenience we reproduce the essentials of the solution in the Appendix.) In this case we assume that $\varphi, \varphi_{0}$ and $\varphi_{1}$ are related by the formula

$$
\varphi=\varphi_{0} \sigma\left(\varphi_{1} / \varphi_{0}\right)
$$

and the necessary and sufficient condition reads $\sigma$ pseudoconcave. In particular, if $\sigma(s)=s^{\eta}(0<\eta<1),(0.3)$ specializes to

$$
\varphi=\varphi_{0}^{1-\eta} \varphi_{1}^{\eta} \text {. }
$$

It will be proved (Proposition 2.3) that in the special case $\varphi_{i}(x)=x^{p}$ $(i=0,1)$ the two conditions $(0.1)$ and $(0.3)$ are essentially equivalent.

The organization of the paper is as follows. In Section 1 and 2 we consider some useful classes of positive functions on $\boldsymbol{R}_{+}^{\times}=(0, \infty)$, in particular, submultiplicative and pseudoconcave functions. (What we - mean by "a little more than concave" is made precise here.) In Section 3 we prove certain variants of two classical inequalities, Carlson's inequality and Khintchine's inequality, which will be needed later. In Section 5 we introduce the Orlicz classes $O^{\varphi}$ and the Orlicz spaces $L^{\varphi}$, after first having treated in the brief Section 4 with the more general case of modular classes and modular spaces. In Section 6 we introduce our interpolation spaces $\langle\vec{A}, \varrho\rangle$. Then, in Section 7 we put all our pieces of information together and, finally, prove our main results (summarized in Theorem 7.3). In Section 8 we consider some concrete cases and in Section 9 a certain limiting case of our main result (where one of the spaces is $L^{\infty}$ ). In Section 10 we prove that in the special case $\varphi_{i}(x)=x^{i}(i=0,1)$ pseudoconcavity of $\varrho$ is, in fact, a necessary condition. Finally, there is the Appendix devoted to the interpolation of Orlicz classes, including weak type interpolation.

1. Submultiplicative functions and the notion of type. We say that two positive functions $\varphi$ and $\psi$ defined in a set $S$ are equivalent if there exists a constant $C$ such that $C^{-1} \varphi(x) \leqslant \psi(x) \leqslant C \varphi(x)$ for $x \in S$. (Occasionally we write $\varphi \approx \psi$.)
In this section and Section 2 we are only concerned with positive functions on $\boldsymbol{R}_{+}^{\times}=(0, \infty)$.

We say that a positive function $s$ on $\boldsymbol{R}_{+}^{\times}$is submultiplicative if

$$
s(x y) \leqslant s(x) s(y), \quad s(1)=1 .
$$

We will need the following classical fact (see e.g. Hille-Phillips [7], p. 244, the additive version):

Proposition 1.1 Let $s$ be submultiplicative. Then $s$ is bounded on any compact set and there exist numbers $p_{0}$ and $p_{1}$ with $p_{0} \leqslant p_{1}$ such that

$$
s(x)=O\left(\max \left(x^{p_{0}}, x^{p_{1}}\right)\right), \quad x \rightarrow 0 \text { or } \infty .
$$

More precisely, if $p_{0}^{*}$ is the supremum of all such $p_{0}$ and $p_{1}^{*}$ the infimum of all $p_{1}$, then the following holds:

$$
\begin{aligned}
& p_{0}^{*}=\sup _{x<1} \log s(x) / \log x=\lim _{x \rightarrow 0} \log s(x) / \log x, \\
& p_{1}^{*}=\inf _{x>1} \log s(x) / \log x=\lim _{x \rightarrow \infty} \log s(x) / \log x .
\end{aligned}
$$

It follows that if instead of (1.2) the sharper inequality

$$
s(x)=o\left(\max \left(x^{p_{0}}, x^{p_{1}}\right)\right), \quad x \rightarrow 0 \text { or } \infty
$$

holds, then $p_{0}<p_{0}^{*}$ and $p_{1}>p_{1}^{*}$, implying, in particular, that we in (1.2) can replace $p_{0}$ and $p_{1}$ with $p_{0}+\varepsilon$ and $p_{1}-\varepsilon$, respectively, provided $\varepsilon>0$ is sufficiently small.

Let now $\varphi$ be a positive function on $\boldsymbol{R}_{+}^{\times}$such that for every $\lambda \epsilon \boldsymbol{R}_{+}^{\times}$ with some constant $C=C(\lambda)$ holds

$$
\varphi(\lambda x) \leqslant C \varphi(x) .
$$

The smallest such $C$ will be denoted by $s_{\varphi}(\lambda)$, i.e.

$$
s_{\varphi}(\lambda)=\sup \varphi(\lambda x) / \varphi(x) .
$$

Clearly, $s_{\varphi}(\lambda)$ is submultiplicative so we may apply Proposition 1.1. Therefore we can replace (1.3) by the more precise

$$
\varphi(\lambda x) \leqslant C \max \left(\lambda^{p_{0}}, \lambda^{p_{1}}\right) \varphi(x)
$$

(with $C$ independent of $\lambda$ ), implying that

$$
s_{\varphi}(\lambda)=O\left(\max \left(\lambda^{p_{0}}, \lambda^{p_{1}}\right)\right) .
$$

We say then that $\varphi$ is of lower type $p_{0}$ and upper type $p_{1}$, or alternatively, more briefly of type $\left(p_{0}, p_{1}\right)$. We sometimes also write $\varphi \in \mathfrak{T}\left(p_{0}, p_{1}\right)$ or, if we want to emphasize the constant $C$ in $(1.5), \varphi \in \mathfrak{T}\left(p_{0}, p_{1} ; C\right)$. 
If (1.6) can be sharpened to

$$
s_{\varphi}(\lambda)=o_{0}\left(\max \left(\lambda^{p_{0}}, \lambda^{p_{1}}\right)\right),
$$

we say that $\varphi$ is lower type $p_{0}+$ and upper type $p_{1}$ - or simply of type $\left(p_{0}+, p_{1}-\right)$ and write $\varphi \in \mathfrak{T}\left(p_{0}+, p_{1}-\right)$ or $\varphi \in \mathfrak{T}\left(p_{0}+, p_{1}-; C\right)$.

Finally, we have the hybrid case where $\left(1.6^{\prime}\right)$ holds only at one endpoint $(0$ or $\infty)$. In an obvious way we then use, for instance, the notation $\mathfrak{I}\left(p_{0}+, p_{1}\right)$. It is also clear what we mean by "of finite upper type", for example.

Clearly, the type is not changed if we replace $\varphi$ by an equivalent function. The following simple fact is often useful.

LEMra 1.1. Every $\varphi \in \mathfrak{T}\left(p_{0}, p_{1} ; O\right)$ is equivalent to some function $\psi \in \mathfrak{I}\left(p_{0}, p_{1} ; \dot{1}\right)$. If $\varphi$ is increasing and $p_{0}>0$ so is $\psi$.

Proof. Take $\psi(x)=\sup \varphi(\lambda x) / \max \left(\lambda^{p_{0}}, \lambda^{p_{1}}\right)$. Then $\varphi \leqslant \psi \leqslant C \varphi$ and $\psi \in \mathfrak{I}\left(p_{0}, p_{1} ; 1\right)$

Remark 1.1. It is not hard to see that if $\varphi \in \mathfrak{T}\left(p_{0}, p_{1} ; 1\right)$ then $\varphi$ is absolutely continuous and we have

$$
p_{0} \leqslant x \varphi^{\prime}(x) / \varphi(x) \leqslant p_{1} \text { a.e. }
$$

Assume now that $\varphi$ is increasing. Then $\varphi$ is automatically of lower type 0 . To ascertain that $\varphi$ is of finite type, it is then sufficient to know that (1.3) holds for $\lambda=2$, i.e. we have

$$
\varphi(2 x) \leqslant C \varphi(x),
$$

which condition often is referred to as the $\Delta_{2}$-condition (see e.g. Krasnoselskii-Rutitskii [9], p. 35).

Assume further that $\varphi$ is continuous with $\varphi\left(\boldsymbol{R}_{+}^{\times}\right)=\boldsymbol{R}_{+}^{\times}$so that $p^{-1}$ exists and is continuous increasing, too. We have then the following simple

LEMma 1.2. If $\varphi$ is of type $\left(p_{0}, p_{1}\right)$ where $p_{0}>0$, then $\varphi^{-1}$ is of type $\left(p_{1}^{-1}, p_{0}^{-1}\right)$.

Proof. By Lemma 1.1, we may assume that $\varphi \in \mathfrak{I}\left(p_{0}, p_{1} ; 1\right)$. We thus have, for instance,

$$
\varphi(\lambda x) \leqslant \lambda^{p_{1}} \varphi(x) \quad \text { if } \lambda>1
$$

which implies with any $\mu$

$$
\lambda^{-1} \varphi(\mu x) \leqslant \varphi^{-1}\left(\lambda^{p_{1}} \mu x\right) .
$$

Taking $\lambda=\mu^{-p_{1}^{-1}}, \mu<1$, now follows

$$
\varphi^{-1}(\mu x) \leqslant \mu^{p_{1}^{-1}} \varphi^{-1}(x) \quad \text { if } \mu<1 .
$$

Thus $\varphi^{-1}$ is of lower type $p_{1}^{-1}$. The corresponding statement with $p_{0}$ is proved in a similar manner.

Another obvious fact which often will be used implicitly is embodied in LEMMA 1.3. If $\varphi \in \mathfrak{I}\left(p_{0}, p_{1}\right), \psi \in \mathfrak{I}\left(q_{0}, q_{1}\right)$, then $q \psi \in \mathfrak{I}\left(p_{0}+q_{0}, p_{1}+q_{1}\right)$.

2. Pseudoconcave functions. We say that a positive function $\varrho$ on $\boldsymbol{R}_{+}^{\times}$is pseudoconcave if it is equivalent to a concave function.

A proof of the following elementary fact can be found in Peetre [15]

Proposition 2.1. $\varrho$ is pseudoconcave if and only if $\varrho \in \mathfrak{T}(0,1)$.

In other words, we have with a suitable $C$ (compare (1.5))

$$
\varrho(\lambda x) \leqslant C \max (1, \lambda) \varrho(x) .
$$

It follows that, with $s_{e}$ defined by (1.4), the following holds

$$
s_{e}(\lambda)=O(\max (1, \lambda))
$$

(compare (1.6)).

The class of functions $\varrho$ satisfying (2.1) will be denoted by $\mathfrak{P}(C)$ $=\mathfrak{I}(0,1 ; C))$. If instead of $(2.2)$,

$$
s_{e}(\lambda)=o(\max (1, \lambda))
$$

holds (compare $\left(1.6^{\prime}\right)$ ), we get instead the class $\mathfrak{P}^{+-}(C)$. Similarly, we define the hybrids $\mathfrak{P}^{+}(C)$ and $\mathfrak{P}^{-}(C)$. If we do not want to specify the constant in $(2.1)$, we write simply $\mathfrak{P}, \mathfrak{P}^{+-}, \mathfrak{P}^{+}, \mathfrak{P}^{-}$.

We now discuss several examples and give some auxiliary remarks. (What is an example or a remark is, of course, an article of faith.)

EXAMPLE 2.1. $\varrho$ belongs to $\mathfrak{P}(1)$ if and only if $\varrho(x)$ is non-decreasing and $\varrho(x) / x$ non-increasing. Clearly, every concave function is in $\mathfrak{P}(\mathbf{1})$. Thus every pseudoconcave function is equivalent to a function in $\mathfrak{\$ ( 1 )}$.

EXAMPLE 2.2. If $\varrho$ is pseudoconcave so is $\varrho^{*}$ defined by $\varrho^{*}(x)=x \varrho(1 / x)$. If $\varrho=\varrho_{\theta}$ denotes the function given by $(0.2)$, we have $\varrho_{\theta}^{*}=\varrho_{1-\theta}$.

Remark 2.1. Example 2.2 displays an important symmetry property of the class $\mathfrak{P}$. The same symmetry is, of course, inherent in all our theory but we do not always point it out explicitly. It is sometimes convenient to introduce the homogeneous function of two variables

$$
R(x, y)=x \varrho(y / x) \quad\left(=y \varrho^{*}(x / y)\right) .
$$

It is now easy to see, for example that $\varrho$ is in $\Re$ (1) if and only if $R$ is non-decreasing in each variable separately, in fact, always in the strong sense we have

$$
x<x^{\prime}, y<y^{\prime} \Rightarrow R(x, y)<R\left(x^{\prime}, y^{\prime}\right) .
$$

To illustrate the usefulness of $R$, we prove the following generalization of Hölder's inequality (the special case $\varrho=\varrho_{0}$ ). 
Proposimion 2.2. Let $\varrho$ be in $\mathfrak{P}(1)$. Then for any two finite positive sequences $\left\{x_{\nu}\right\}$ and $\left\{y_{\nu}\right\}$

$$
\sum R\left(x_{v}, y_{v}\right) \leqslant 2 R\left(\sum x_{\nu}, \sum y_{v}\right)
$$

holds with $R$ defined by (2.3).

Proof. Write $x_{\nu}=\xi_{v} X, y_{\nu}=\eta_{\nu} Y$ with $\sum \xi_{v}=1, \sum \eta_{\nu}=1$. Then we get

$$
\sum R\left(x_{v}, y_{v}\right) \leqslant \sum \max \left(\xi_{v}, \eta_{\nu}\right) R(X, Y) \leqslant\left(\sum \xi_{\nu}+\sum \eta_{\nu}\right) R(X, Y)
$$

Since $X=\sum x_{v}, Y=\sum y_{v}$, this proves (2.4).

EXAMPLE 2.3. If $\varrho(x)=\left(\varrho\left(x^{c}\right)\right)^{1 / c}$ for some $c \neq 0$ and $\varrho \in \mathfrak{P}$, then $\varrho \in \mathfrak{P}$. Olearly, we have $s_{\varrho}^{-}(\lambda)=\left(s_{\varrho}\left(\lambda^{c}\right)\right)^{1 / c}$.

EXAMPLE 2.4. The function $\varrho=\varrho_{\theta \alpha \beta}$, given by $\left(0.2^{\prime}\right)$, is in $\mathfrak{P}^{+-}$for all values of the parameters $(0<\theta<1, \alpha, \beta$ real $)$. Note that $\varrho_{\theta \alpha \beta}^{*}=\varrho_{1-\theta, \beta \alpha}$. To see this, we apply Lemma 1.3 together with Example 2.2 and the fact that $s_{\varrho}=O(\varrho)$ if $\varrho=\log (e+x)$.

Remark 2.2. There is an obvious relation between the classes $\mathfrak{T}$ and $\mathfrak{P}: \varphi$ is in $\mathfrak{I}\left(p_{0}, p_{1}\right)$ if and only if there exists a function $\sigma$ in $\mathfrak{P}$ such that (2.5)

$$
\varphi(x)=x^{p_{0}} \sigma\left(x^{p_{1}-p_{0}}\right) .
$$

We can use this to settle the question of equivalence of conditions $(0.1)$ and $(0.3)$ in the special case $\varphi_{i}(t)=t^{p_{i}}(i=0,1)$, mentioned in the introduction. We formulate this as

Proposition 2.3. Let $\sigma$ be a continuous increasing function with $\sigma\left(\boldsymbol{R}_{+}^{\times}\right)$ $=\boldsymbol{R}_{+}^{\times}$. Define $\varphi$ by $(2.5)$, with $0<p_{0}<p_{1}$. Then $\varphi$ is continuous increasing with $\varphi\left(\boldsymbol{R}_{+}^{\times}\right)=\boldsymbol{R}_{+}^{\times}$so $\varphi^{-1}$ exists. Define $\varrho$ by

$$
\varphi^{-1}(x)=x^{1 / p_{0}} \varrho\left(x^{1 / p_{1}-1 / p_{0}}\right)
$$

Then $\varrho$ is in $\mathfrak{P}$ if and only if $\sigma$ is in $\mathfrak{P}$.

Proof. The first statement concerning the existence of $\varphi^{-1}$ is elementary, Assume now that $\sigma$ is in $\mathfrak{P}$. Then, by the preceding remark, $\varphi$ is in $\mathfrak{I}\left(p_{0}, p_{1}\right)$. Thus, by Lemma $1.2, p^{-1}$ is in $\mathfrak{I}\left(p_{1}^{-1}, p_{0}^{-1}\right)$. Applying the same remark with (2.6), now follows $\varrho \in \mathfrak{P}$. The converse is obtained if we reverse the role of $\varrho$ and $\sigma$.

3. Generalization of two classical inequalities. We begin by discussing an inequality of Carlson type. (Regarding Carlson's inequality and its generalizations see Beckenbach-Bellman [1], pp. 175-177 and p. 186.)

Proposition 3.1. Let $1 \leqslant p<\infty$. Let $\varrho$ be a positive measurable funotion of finite type and such that, in addition,

$$
\left(\int_{0}^{\infty}(\min (1, x / t) \varrho(t))^{p^{\prime}} d t / t\right)^{1 / p^{\prime}} \leqslant C \varrho(x), \quad 1 / p+1 / p^{\prime}=1
$$

holds. Let $\left\{u_{\nu}\right\}$ be a finite positive sequence. Then

$$
\sum u_{\nu} \leqslant C R\left(\left(\sum\left(u_{\nu} / \varrho\left(2^{\nu}\right)\right)^{p}\right)^{1 / p},\left(\sum\left(2^{\nu} u_{\nu} / \varrho\left(2^{\nu}\right)\right)^{p}\right)^{1 / p}\right)
$$

with $R$ defined by (2.3).

Proof. Put

$$
U=\left(\sum\left(u_{v} / \varrho\left(2^{v}\right)\right)^{p}\right)^{1 / p}, \quad V=\left(\sum\left(2^{v} u_{\nu} / \varrho\left(2^{v}\right)\right)^{p}\right)^{1 / p} .
$$

Then using (3.1) and Hölder's inequality we get with any $x$

$$
\begin{aligned}
\sum u_{\nu} & =\sum_{2^{\nu} \leqslant x} u_{\nu}+\sum_{2^{\nu}>x} u_{\nu} \leqslant\left(\sum_{2^{\nu} \leqslant x}\left(\varrho\left(2^{\nu}\right)\right)^{p^{\prime}}\right)^{1 / p^{\prime}} U+\left(\sum_{2^{\nu}>x}\left(\varrho\left(2^{\nu}\right) / 2^{\nu}\right)^{p^{\prime}}\right)^{1 / p^{\prime}} V \\
& \leqslant C \varrho(x) U+C(\varrho(x) / x) \nabla=C(U+\nabla / x) \varrho(x) .
\end{aligned}
$$

Taking $x=\nabla / U$, we obtain

$$
\sum u_{v} \leqslant C U \varrho(\nabla / U)=C R(U, \nabla) \text {, }
$$

which is the desired inequality (3.2).

Condition (3.1) is certainly fulfilled if the function $s_{e}$ (defined as by (1.4)) satisfies

$$
\left(\int_{0}^{\infty}\left(\min (1,1 / \lambda) s_{e}(\lambda)\right)^{p^{\prime}} d \lambda / \lambda\right)^{1 / p^{\prime}}<\infty .
$$

Assume now that $\varrho \in \mathfrak{P}$. If $p=1$, then (3.4) is substantially just (2.1). But if $p>1,(3.4)$ is, in fact, equivalent to $\left(2.2^{\prime}\right)$, i.e. $\varrho \in \mathfrak{P}^{+-}$. Indeed, in view of Proposition 1.1, it is plain that $\left(2.2^{\prime}\right)$ implies (3.1). For the converse, we write (2.1) in the form

$$
\varrho(t) \geqslant C \min (1, t / \lambda x) \varrho(\lambda x) \text {. }
$$

Inserting this in (3.1), we readily obtain $\left(2.2^{\prime}\right)$. In particular, we have thus proved

COROLLARY 3.1. (3.2) is applicable with $p=1$ if $\varrho € \mathfrak{P}$, and with any $p(1 \leqslant p \leqslant \infty)$ if $\varrho \in \mathfrak{P}^{+-}$.

EXAMPLE 3.1. In particular, (3.2) is applicaible with any $p$ if $\varrho=\varrho_{\theta \alpha \beta}$. This follows at once from Example 2.4.

Remark 3.1. If instead $\varrho \epsilon \mathfrak{P}^{+}$, we can, in a similar way, prove

$$
\sum u_{\nu} \leqslant C R\left(\left(\sum\left(u_{\nu} / \varrho\left(2^{\nu}\right)\right)^{p}\right)^{1 / p}, \sum 2^{p} u_{\nu} / \varrho\left(2^{\nu}\right)\right) .
$$

(This will be useful in Section 9.)

We also mention the following variant of Proposition 3.1: 
Proposition 3.2. Let $1 \leqslant p \leqslant \infty$. Let $\varphi, \varphi_{0}$ and $\varphi_{1}$ be continuous increasing functions with $\varphi$ expressed in terms of $\varphi_{0}$ and $\varphi_{1}$ with the aid of $(0.1)$. If $p=1$, assume that $\varrho \in \mathfrak{P}$, otherwise $\varrho \in \mathfrak{P}^{+-}$. Assume also that $\varphi$ is of finite upper type. Let $\left\{u_{\nu}\right\}$ be a finite positive sequence. Then the following holds :

(3.5) $\quad p\left(\sum u_{\nu}\right) \leqslant O\left(\varphi_{0}\left(\left(\sum\left(u_{\nu} / \varrho\left(2^{\nu}\right)\right)^{p}\right)^{1 / p}\right)+\varphi_{1}\left(\left(\sum\left(2^{\nu} u_{\nu} / \varrho\left(2^{\nu}\right)\right)^{p}\right)^{1 / p}\right)\right)$.

Proof. Define $U, V$ by (3.3) and put further $x=\varphi_{0}(U)+\varphi_{\mathbf{1}}(V)$. Then it follows from (3.2) and Remark 2.1 that

$$
\sum u_{\nu} \leqslant C R(U, V) \leqslant O R\left(\varphi_{0}^{-1}(x), \varphi_{1}^{-1}(x)\right) .
$$

Now (0.1) may be rewritten as

$$
\varphi^{-1}=R\left(\varphi_{0}^{-1}, \varphi_{1}^{-1}\right)
$$

Therefore,

$$
\sum u_{v} \leqslant C \varphi^{-1}(x)
$$

or

$$
\varphi\left(\sum u_{\nu}\right) \leqslant \varphi\left(O \varphi^{-1}(x)\right) \leqslant C x
$$

by (1.3).

It is convenient to insert here the following result which clarifies the assumptions made on $\varphi$ in Proposition 3.2. (This will be needed in Section 7).

Propositron 3.3. Let $\varphi_{0}$ and $\varphi_{1}$ be continuous increasing functions on $\boldsymbol{R}_{+}^{\times}$with $\varphi_{i}\left(\boldsymbol{R}_{+}^{\times}\right)=\boldsymbol{R}_{+}^{\times}(i=0,1)$. Let $\varrho$ be in $\mathfrak{\beta}(1)$. Define $\varphi$ by $(0.1)$ (or equivalently (3.6)). If $\varphi_{0}$ and $\varphi_{1}$ have finite upper type so has $\varphi$. Also if $\varphi_{0}$ and $\varphi_{1}$ have positive lower type so has $\varphi$.

Proof. That $\varphi$ is well-defined follows from Remark 2.1, if we again rewrite (0.1) as (3.6). There exists a number $p>1$ such that $p_{0}$ and $p_{1}$ are both in $(1, p)$. Then, by Lemma $1.2, \varphi_{0}^{-1}$ and $\varphi_{1}^{-1}$ are both in $\mathfrak{I}\left(p^{-1}, 1\right)$ From (3.6) it follows once more using Remark 2.1 that $\varphi^{-1}$ is also in $\mathfrak{T}\left(p^{-1}, 1\right)$. Appealing again to Lemma 1.2 , we find $p \in \mathfrak{I}(1, p)$. The second statement concerning the lower type is proved in an analogous fashion.

We now pass to our second topic, a generalization of the classical Khintchine's (or Littlewood's) inequality.

Proposition 3.4. Let $\left\{u_{v}\right\}$ be any finite real sequence, and consider random sums $s=\sum \pm u_{\nu}$. Put also $m=\left(\sum u_{\nu}^{2}\right)^{1 / 2}$. Let $\varphi$ be any positive non-decreasing function on $\boldsymbol{R}_{+}=[0, \infty)$ whose restriction to $\boldsymbol{R}_{+}^{\times}=(0, \infty)$ satisfies $\left(1.3^{\prime}\right)$. Then there exists a constant $C$, depending on $C$ in $\left(1.3^{\prime}\right)$ only, such that

$$
C^{-1} \varphi(m) \leqslant \mathbf{E}(\varphi(|s|)) \leqslant C \varphi(m),
$$

where $\mathrm{E}$ stands for "expectation".

Proof. We take for granted the classical fact that the result is true for $\varphi(x)=x^{p}, p>0$ (see e.g. Zygmund [18], Vol: I, p. 213), i.e.

$$
C^{-1} m^{p} \leqslant \mathrm{E}\left(|s|^{p}\right) \leqslant C m^{p}
$$

(with $C$ depending on $p$ only).

(a) We prove the second inequality (3.7) first. By Proposition 2.1 and Remark 2.2 upon passing to an equivalent function, we may as well assume that $\varphi$ is of the form $\varphi(x)=\psi\left(x^{p}\right)$, where $\psi$ is a concave function and $p>0$ suitable. It follows that $\varphi$ is given by the formula

$$
\varphi(x)=\inf _{(A, B) \in H}\left(A x^{p}+B\right),
$$

where $H$ is a suitable subset of the octant $\boldsymbol{R}_{+} \times \boldsymbol{R}_{+}$. In particular, for every $(A, B) \epsilon H$ we thus have, by $(3.9)$,

This implies

$$
\varphi(|s|) \leqslant A|s|^{p}+B \text {. }
$$

$$
\mathrm{E}(\varphi(|s|)) \leqslant A \mathrm{E}\left(|s|^{p}\right)+B \leqslant C\left(A m^{p}+B\right),
$$

where we have used the second inequality (3.8). Appealing once more to (3.9), we clearly get the desired result.

(b) There remains the first inequality (3.7). Let us put $\Phi(x)=x^{p} / \varphi(x)$.

Then $\Phi$ is of the form $\Phi(x)=\Psi\left(x^{p}\right)$ with $\Psi \in \mathfrak{P}(1)$. Therefore the result of (a) is applicable to $\Phi$. Write now

$$
|s|^{p / 2}=\sqrt{\varphi(|s|)} \sqrt{\Phi(|s|)} .
$$

Hölder's inequality gives

$$
\mathrm{E}\left(|s|^{p / 2}\right) \leqslant \sqrt{\mathbf{E}(\varphi(|s|))} \sqrt{\mathbf{E}(\Phi(|s|))}
$$

But in view of (a),

$$
\mathrm{E}(\Phi(|s|)) \leqslant C m^{p} / \varphi(m)
$$

and the first inequality (3.8) gives

$$
C^{-1} m^{p / 2} \leqslant \mathrm{E}\left(|s|^{p / 2}\right) .
$$

Inserting these estimates in (3.10), we again arrive at the desired result

Remark 3.2. (1.3') is, in fact, necessary for the second inequality (3.7) to hold (but not the first). Indeed, consider the sequence $\left\{u_{1}, u_{2}\right\}$, then

$$
\varphi\left(\left|u_{1}+u_{2}\right|\right)+\varphi\left(\left|u_{1}-u_{2}\right|\right) \leqslant C \varphi\left(\sqrt{u_{1}^{2}+u_{2}^{2}}\right) .
$$


Take, in particular, $u_{1}=u_{2}=1 / \sqrt{2} x$. Then it follows that

$$
\varphi(\sqrt{2} x) \leqslant \dot{C} \varphi(x)
$$

or upon iteration (1.3').

4. On modulars. We first nail down our terminology concerning topological vector spaces (cf. e.g. Köthe [8]).

As is well-known a topological vector space is metrizable if and only if there exists a denumerable basis of neighborhoods of 0 (for the proof see also Gustavsson [6]). A complete metrizable vector space will be called an F-space. As is likewise well known, a topological vector space is quasinormable if and only if it is locally bounded. A complete quasi-normed. space will be called a quasi-Banach space.

Now we give a rapid introduction to the theory of modulars, mainly following Musielak-Orlicz [11].

Let $E$ be any vector space (over $R$, to fix the ideas). By a modular in $E$ we mean an extended real-valued function $\|a\|$ in $E$ such that

$$
\begin{gathered}
\|a\|=0 \Leftrightarrow a=0, \\
\|\lambda a\| \leqslant\|a\| \quad \text { if } \quad|\lambda| \leqslant 1,\|-a\|=\|a\|, \\
\lim _{\lambda \rightarrow 0}\|\lambda a\|=0 \quad \text { if } \quad\|a\|<\infty,
\end{gathered}
$$

(4.4) $\quad\|(a+b) / h\| \leqslant k(\|a\|+\|b\|) \quad$ for certain constants $h$ and $k$.

Some distinguished special cases of (4.4) are:

$h=1$

quasi-F-norm,

$h=1, k=1 \quad$ F-norm,

$h=2, k=1 \quad$ essentially a modular in the sense of Musielak-Orlicz [11].

A quasinorm is a quasi-F-norm such that (4.2) can be replaced by the sharper condition

$$
\|\lambda a\|=|\lambda|\|a\| .
$$

If we drop condition (4.1), we use the suffix "semi", speaking thus of a semi-quasi-F-norm, etc.

Given a modular $\|a\|$ in $E$, we denote by $\bar{E}$ (modular class) the subset of $E$ determined by the condition $\|a\|<\infty$. A sufficient condition for $\bar{E}$ to be a subspace is that $\|a\|$ is a quasi-F-norm.

We denote by $\bar{E}^{*}$ (modular space) the subspace determined by the condition $\|a / a\|<\infty$ for some $\alpha$ (depending on $a$ ), i.e. $\bar{E}^{*}=\bigcup_{\lambda>0} \lambda \bar{E}$. In $\bar{E}^{*}$ we have a natural topology, if we use as a basis for neighborhoods of 0 the sets $U_{a \beta}=\left\{\left.a\right|^{\prime}\|a / \alpha\| \leqslant \beta\right\}$ with $\alpha, \beta>0$. (From (4.4) readily follows the crucial property $U_{\alpha \beta}+U_{\alpha \beta} \subset U_{h \alpha, 2 k \beta}$.) Further we assume that this topology is Hausdorff. Since we can, in fact, do with a denumerable number of $U_{a \beta}$, we know thus that $\bar{B}^{*}$ is metrizable. An explicit quasi-F-norm is given by

$$
\text { (4.5) } \quad\|a\|^{*}=\inf \left\{\alpha\left|\|a \mid \alpha\| \leqslant \alpha^{r}\right\},\right.
$$

where $r \geqslant 0$ has to be chosen so that $2 k \leqslant h^{r}$. We now ask when $\bar{E}^{*}$ is locally bounded. A necessary and sufficient condition is that we can find an $U_{\alpha_{0} \beta_{0}}$ such that for all $U_{\alpha \beta}$ there exists $\lambda>0$ such that $\lambda U_{a_{0} \beta_{0}} \subset U_{\alpha \beta}$. With no essential loss of generality we may assume that $\alpha_{0}=\beta_{0}=1$. We then readily find the condition

$$
\|a\| \leqslant 1 \Rightarrow\|\lambda a\| \leqslant \frac{1}{2} k \text {. }
$$

If (4.6) is fulfilled, we can take $r=0$ in (4.5), i.e., we have the quasinorm $\left(4.5^{\prime}\right)$

$$
\|a\|^{*}=\inf \{\alpha \mid\|a / \alpha\| \leqslant 1\} .
$$

We conclude by the following simple result which says something about linear operators in modular spaces.

Proposirion 4.1. Let $E$ and $F$ be vector spaces equipped with modulars, and consider a linear mapping $T: E \rightarrow F$. Assume that $T(\bar{E}) \subset \bar{F}$ and that

$$
\|T a\| \leqslant C_{1}\|a\|+C_{2}, \quad a \in \bar{E},
$$

for some $C_{1}, C_{2}$. Then $T\left(\bar{E}^{*}\right) \subset \bar{F}^{*}$. If the set $\nabla=\left\{b \mid\|b\| \leqslant C_{1}+C_{2}\right\}$ is bounded in $\bar{F}^{*}$ (which implies that $\bar{F}^{*}$ is locally bounded), we have

$$
\text { (4.8) } \quad\|T a\|^{*} \leqslant C_{3}\|a\|^{*}, \quad a \in \bar{E}^{*},
$$

with $\|\cdot\|^{*}$ defined in accordance with $\left(4.5^{\prime}\right)$ and with a suitable $C_{3}$.

Proof. By the boundedness of $V$, we can determine $C_{3}$ such that (cf. (4.6))

$$
\|b\| \leqslant C_{1}+C_{2} \Rightarrow\left\|b / C_{3}\right\| \leqslant 1 \text {. }
$$

take now $a \in \bar{E}^{*}$ with $\|a\|^{*} \leqslant 1$. From (4.7) follows then, for any a subject $0\|a / a\| \leqslant 1$,

$$
\left\|T a_{1} / \alpha\right\| \leqslant C_{1}\|a / \alpha\|+C_{2} \leqslant C_{1}+C_{2}
$$

so that, by (4.9), $\left\|T a / C_{3} a\right\| \leqslant 1$. Upon taking the inf, (4.8) readily follows.

Remark 4.1. In particular, $T$ in Proposition 4.1 is continuous (when restricted to $\bar{B}^{*}$ ). Conversely, if $\bar{B}^{*}$ is a'so locally bounded, every continuous linear mapping T: $\bar{E}^{*} \rightarrow \bar{F}^{*}$ satisfies (4.8) (but, of course, not (4.7) in general). The least constant $C_{3}$ in (4.8) is the operator quasinorm of $T$.

5. Introduction of Orlicz spaces. For a general discussion of Orlicz spaces in the classical case where the function $\varphi$ below is convex, see Krasnoselskii-Rutitskii [9]. 
Let $M$ be a measure space equipped with a positive measure $\mu$. Let $\varphi$ be a continuous function on $\boldsymbol{R}_{+}$with $\varphi(0)=0$ and whose restriction to $\boldsymbol{R}_{+}^{*}$ is increasing (and thus positive.) We apply the general framework. of the previous section to the case where $E$ is the space of all $\mu$-measurable functions $a$ on $M$, taking

$$
\|a\|=\|a\|_{O^{\varphi}}=\int_{M} \varphi(|a|) d \mu .
$$

Clearly, (5.1) defines a modular with $h=2, l=1$. We set $\bar{E}=O^{\varphi}$ (Orlicz class) and $\bar{E}^{*}=\left(0^{\varphi}\right)^{*}=L^{\varphi}$ (Orlicz space). If $\varphi$ has finite upper type (i.e. satisfies the $\Delta_{2}$-condition $\left(1.3^{\prime}\right)$ ), then $O^{\varphi}$ is in fact an F-space, since completeness can be readily demonstrated. If $\varphi$ has positive lower type, then (4.6) is applicable so that $L^{\varphi}$ is locally bounded. Again we have completeness so that $L^{\varphi}$ is a quasi-Banach space with the quasinorm (cf. $\left(4.5^{\prime}\right)$ )

$$
\|a\|^{*}=\|a\|_{L^{p}}=\inf \left\{\alpha \mid \int_{M} \varphi(|a| / a) d \mu \leqslant 1\right\}
$$

In particular, Proposition 4.1 is then applicaible to $L^{\varphi}$.

As a preparation for Section 7 we include now the following simple result:

Proposition 5.1. Let $\varphi, \varphi_{0}$ and $\varphi_{1}$ be continuous increasing functions on $\boldsymbol{R}_{+}$, with $p$ of positive lower type and expressed in terms of $\varphi_{0}$ and $\varphi_{1}$ by the aid of (0.1), with e pseudoconcave. Assume that

$$
\int_{M} \varphi_{i}\left(\left|a_{i}\right|\right) d \mu \leqslant C_{i}(i=0,1) \quad \text { and } \quad|a| \leqslant\left|a_{0}\right| \varrho\left(\left|a_{1}\right| /\left|a_{0}\right|\right) .
$$

Then holds

$$
\int_{M} \varphi(|a|) d \mu \leqslant C
$$
we get

Proof. Put $b_{i}=p_{i}\left(\left|a_{i}\right|\right)(i=0,1), b=b_{0}+b_{1}$. Using Remark 2.1,

$$
|a| \leqslant R\left(\varphi_{0}^{-1}\left(b_{0}\right), \varphi_{1}^{-1}\left(b_{1}\right)\right) \leqslant C R\left(\varphi_{0}^{-1}(b), \varphi_{1}^{-1}(b)\right)=C p^{-1}(b)
$$

or

$$
\varphi(|a| / C) \leqslant \varphi_{0}\left(\left|a_{0}\right|\right)+\varphi_{1}\left(\left|a_{1}\right|\right) .
$$

Invoking the positive lower type of $p$ and integrating, we readily get the desired result.

Remark 5.1. Proposition 5.1 can be given the following inter pretation.

Let $A_{0}$ and $A_{1}$ be any two rearrangement invariant quari-Banach spaces of $\mu$-measurable functions on $M$ and let $\varrho$ be pseudoconcave. We ntroduce $A=A_{0} \varrho\left(A_{1} / A_{0}\right)\left(=R\left(A_{0}, A_{1}\right)\right)$ to be the space of those $\mu$-mea- surable functions $a$ for which one can find a constant $C$ and functions $a_{0} \in A_{0}$ and $a_{1} \in A_{1}$ such that

(5.3) $\quad|a| \leqslant C\left|a_{0}\right| \varrho\left(\left|a_{1}\right| /\left|a_{0}\right|\right) \quad\left(=C R\left(\left|a_{0}\right|,\left|a_{1}\right|\right)\right), \quad\left\|a_{0}\right\|_{A_{0}} \leqslant 1,\left\|a_{1}\right\|_{A_{1}} \leqslant 1$.

We equip $A$ with the quasinorm $\|a\|_{A}=\inf C$, where $C$ runs over all constants occuring in (5.3). (That this is a quasinorm is easy to see; for instance, the quasitriangle inequality results from application of Proposition 2.2.) Then $A$ becomes a quasi-Banach space. If $\varrho=\varrho_{\theta}$, we obtain as a special case the space $A_{0}^{1-\theta} A_{1}^{\theta}$ of Calderón [4]. Now take $A_{i}=L^{\varphi_{i}}(i=0,1)$ as in Proposition 5.1. Then we see that

$$
L^{\varphi_{0}} \varrho\left(L^{\varphi_{1}} / L^{\varphi_{0}}\right) \subset L^{\varphi},
$$

the embedding being continuous.

6. A general interpolation method. Let $\vec{A}=\left\{A_{0}, A_{1}\right\}$ be any quasiBanach couple (i.e. $A_{0}$ and $A_{1}$ are quasi-Banach spaces, both continuously embedded in a Hausdorff topological vector space $\mathscr{A}$ ) and let $\varrho$ be a pseudoconcave function ( $\varrho € \mathfrak{P})$. Then we denote by $\langle\vec{A}, \varrho\rangle=\left\langle A_{0}, A_{1}, \varrho\right\rangle$ the space of elements $a \in \Sigma(\vec{A})=A_{0}+A_{1}$ (the hull of $A_{0}$ and $A_{1}$ in $\mathscr{A}$ ) such that there is a sequence $u=\left\{u_{\nu}\right\}_{v \in Z}$ of elements $u_{\nu} \in \Delta(\vec{A})=A_{0} \cap A_{1}$ (the intersection of $A_{0}$ and $A_{1}$ in $\mathscr{A}$ ) such that

$$
\left.a=\sum_{\nu \in \mathbb{Z}} u_{\nu} \text { (convergence in } \Sigma(\vec{A}) !\right) \text {, }
$$

(6.2) for every finite subset $F \subset \boldsymbol{Z}$ and every real sequence $\xi=\left\{\xi_{\nu}\right\}_{v \in F}$ with $\left|\xi_{v}\right| \leqslant 1$ we have $\left\|\sum \xi_{v} u_{v} / \varrho\left(2^{v}\right)\right\|_{\Delta_{0}} \leqslant C$, $\left\|\sum \xi_{\nu} 2^{v} u_{\nu} / \varrho\left(2^{v}\right)\right\|_{A_{1}} \leqslant C$ with $C$ independent of $F$ and $\xi$.

Example 6.1. If $\varrho=\varrho_{\theta}$, we obtain the spaces denoted by $\langle\vec{A}\rangle_{\theta}$ in Peetre [13]

We equip $\langle\vec{A}, \varrho\rangle$ with the semi-quasinorm

$$
\|a\|_{\langle\vec{A}, \varrho\rangle}=\inf _{u} C
$$

where the inf is taken over all sequences $u$ permissible in (6.1) and (6.2) (with $O$ having the same significance as in (6.2)).

Remark 6.1. If we replace $\varrho$ by an equivalent (pseudoconcave) function, we get the same space but the semi-quasinorm is, in general, replaced by an equivalent one.

Remark 6.2. If $\varrho \in \mathfrak{B}^{+-}$, then it is possible to show that $\|a\|_{\langle\vec{A}, e\rangle}$ is indeed a quasinorm and that $\langle\vec{A}, \varrho\rangle$ is a quasi-Banach space. In what follows only this case will matter. 
Now we establish the following basic fact:

Proposition 6.1. Let $\vec{A}=\left\{A_{0}, A_{1}\right\}$ and $\vec{B}=\left\{B_{0}, B_{1}\right\}$ be any two quasi-Banach couples. If $T: \vec{A} \rightarrow \vec{B}$ is any continuous linear mapping (meaning that $T_{i}=T \mid A_{i}: A_{i} \rightarrow B_{i}(i=0,1)$ is a continuous linear mapping in the ordinary sense), then $T:\langle\vec{A}, \varrho\rangle \rightarrow\langle\vec{B}, \varrho\rangle$ (in a similar sense $)$ and we have

$$
\|T\|_{\langle\vec{A}, Q\rangle,\langle\vec{B}, \varrho\rangle} \leqslant \max \left(\|T\|_{\mathcal{A}_{0}, B_{0}},\|T\|_{\mathcal{A}_{1}, B_{1}}\right)
$$

for the operator quasinorms involved (cf. Remark 4.1). In other words, the functor $\vec{A} \mapsto\langle\vec{A}, \varrho\rangle$ is an interpolation space. More precisely, if $s_{Q}$ is defined by (1.4), we have

$$
\|T\|_{\langle\vec{A}, e\rangle,\langle\vec{B}, e\rangle} \leqslant C\|T\|_{\mathcal{A}_{0}, B_{0}} s_{\varrho}\left(\|T\|_{\mathcal{A}_{1}, B_{1}} /\|T\|_{\mathcal{A}_{0}, B_{0}}\right) .
$$

Thus we have an interpolation space of type $s_{Q}$.

Proof. Let $a \in\langle\vec{A}, \varrho\rangle$ with $\|a\|_{\langle\vec{A}, \varrho\rangle}<1$. Choose $u=\left\{u_{\nu}\right\}_{\nu \in Z}$ satisfying (6.1) and (6.2), with $C=1$. Define $v=\left\{v_{v}\right\}_{\nu \in Z}$ by $v_{v}=T u_{v+x}$, where $x \in \boldsymbol{Z}$ is at our disposal. Then we have the analogue of (6.1) with $T a$ and $v$ :

$$
T a=\sum_{p \in Z} v_{\nu}
$$

If $\eta=\left\{\eta_{v}\right\}_{\nu \in F}$ is any real sequence with $\left|\eta_{v}\right| \leqslant 1$, writing

$$
M_{i}=\|T\|_{\mathcal{A}_{i}, B_{i}} \quad(i=0,1), \quad \xi_{\nu}=\eta_{\nu-\varkappa} \varrho\left(2^{\nu}\right) / \varrho\left(2^{\nu-\varkappa}\right),
$$

we also get

$$
\begin{gathered}
\left\|\sum \eta_{\nu} v_{\nu} / \varrho\left(2^{v}\right)\right\|_{B_{0}} \leqslant M_{0}\left\|\sum \eta_{\nu} u_{v+x} / \varrho\left(2^{\nu}\right)\right\|_{A_{0}}=M_{0}\left\|\sum \xi_{\nu} u_{\nu} / \varrho\left(2^{\nu}\right)\right\|_{A_{0}}, \\
\left\|\sum \eta_{\nu} 2^{v} v_{v} / \varrho\left(2^{v}\right)\right\|_{B_{1}} \leqslant M_{1}\left\|\sum \eta_{\nu} 2^{v} u_{\nu+x} / \varrho\left(2^{v}\right)\right\|_{A_{1}} \\
=M_{1} 2^{-x}\left\|\sum \xi_{\nu} 2^{v} u_{\nu} / \varrho\left(2^{v}\right)\right\|_{A_{1}}
\end{gathered}
$$

In particular, if $x=0$, we have $\xi_{\nu}=\eta_{v}$. Therefore, we get from $(6.2)$ (with $C=1$ )

$$
\left\|\sum \eta_{\nu} v_{\nu} / \varrho\left(2^{v}\right)\right\|_{B_{0}} \leqslant M_{0}, \quad\left\|\sum \eta_{\nu} 2^{v} v_{\nu} / \varrho\left(2^{\nu}\right)\right\|_{B_{1}} \leqslant M_{1} .
$$

This is the corresponding analogue of (6.2). This proves $T a \epsilon\langle\vec{B}, \varrho\rangle$ and also the inequality $(6.4)$. In the general case we get instead

$$
\begin{gathered}
\left\|\sum \eta_{\nu} v_{v} / \varrho\left(2^{\nu}\right)\right\|_{B_{0}} \leqslant M_{0} s_{e}\left(2^{x}\right), \\
\left\|\sum \eta_{\nu} 2^{v} v_{v} / \varrho\left(2^{\nu}\right)\right\|_{B_{1}} \leqslant M_{1} 2^{-x} s_{Q}\left(2^{x}\right) .
\end{gathered}
$$

Choosing now $2^{*} \approx M_{1} / M_{0}$, we have the proof of (6.5).
7. Application to interpolation of Orlicz spaces. Let $\varphi_{0}$ and $\varphi_{1}$ be continuous increasing functions on $\boldsymbol{R}_{+}^{\times}$such that $\varphi_{i}\left(\boldsymbol{R}_{+}^{\times}\right)=\boldsymbol{R}_{+}^{\times}$ $(i=0,1)$. Let $\varrho$ be in $\mathfrak{P}(1)$ and define $\varphi$ by $(0.1)$. If both $\varphi_{0}$ and $\varphi_{1}$ have positive lower type so has $\varphi$ (Proposition 3.3). Then $L^{\varphi}, L^{\varphi_{0}}$ and $L^{\varphi_{1}}$ are all quasi-Banach spaces. (An analogous statement holds with finite upper type.) We would like to prove that the Orlicz space $L^{\varphi}$ is an interpolation space with respect to the "Orlicz (space) couple" $\vec{L}^{\varphi}=\left\{L^{\varphi_{0}}, L^{\varphi_{1}}\right\}$ (with $\vec{\varphi}=$ $\left.\left(\varphi_{0}, \varphi_{1}\right)\right)$. Here is our first result in this sense.

THeOREM 7.1. Assume that both $\varphi_{0}$ and $\varphi_{1}$ have positive lower type and that one of them, say $\varphi_{0}$, has finite upper type (i.e. satisfies the $\Delta_{\mathbf{2}^{-}}$-condition $\left.\left(1.3^{\prime}\right)\right)$. Assume also that $\varrho \in \mathfrak{P}(1)$. Then with $\varphi$ given by $(0.1), L^{\varphi} \subset\left\langle\overrightarrow{L^{\varphi}}, \varrho\right\rangle$, continuous embedding.

Proof. Let $a \in L^{p}$. With no loss of generality we may assume that $\|a\|_{L^{\varphi}} \leqslant 1$. Thus we have $\int_{\dot{I}} \varphi(|a|) d \mu \leqslant 1$. For fixed $z$ let us consider the
function

$$
\psi(t)=\varphi_{0}(z / \varrho(t))-\varphi_{1}(t z / \varrho(t)) .
$$

It is easy to see that $\psi$ is continuous decreasing and that

$$
\lim _{t \rightarrow 0} \psi(t)>0, \quad \lim _{t \rightarrow \infty} \psi(t)<0 .
$$

Thus there exists a unique $t$ such that $\psi(t)=0$. We denote this $t$ by $h(z)$. Then $h$, clearly, is a continuous function. From $(0.1)$ now follows that

Indeed, writing

$$
\varphi(z)=\varphi_{0}(z / \varrho(t))=\varphi_{1}(t z / \varrho(t)) .
$$

we have

$$
x=z / \varrho(t), \quad y=t z / \varrho(t),
$$

so that (0.1) gives

$$
\varphi^{-1}\left(\varphi_{0}(x)\right)=x \varrho(y / x)=x \varrho(t)=z, \quad \varphi(z)=\varphi_{0}(x)
$$

and proves (7.1.). For $\nu_{\in} \boldsymbol{Z}$ we put

$$
e_{v}=\left\{m|m \in M,| a(m) \mid \epsilon h^{-1}\left(\left[2^{v}, 2^{v+1}\right)\right)\right\} .
$$

Since $h$ is continuous, $e_{\nu}$ is $\mu$-measurable. Moreover,

$$
M=\bigcup_{\nu \in \mathbb{Z}} e_{\nu}, \quad e_{\nu} \cap e_{\varkappa}=\emptyset(\nu \neq x) .
$$

Therefore, putting

$$
u_{v}= \begin{cases}a & \text { on } e_{v} \\ 0 & \text { otherwise }\end{cases}
$$


the sequence $\left\{u_{v}\right\}_{v \in Z}$ will satisfy (6.1). There remains the verification of (6.2). Let us estimate first $\left\|\sum \xi_{v} u_{p} / \varrho\left(2^{\nu}\right)\right\|_{L^{\varphi_{0}}}$. Using (7.1) and (1.3') (with $\varphi_{0}$ ), we get

$$
\begin{aligned}
& \int_{M} \varphi_{0}\left(\mid \sum_{\nu \in F^{\prime}} \xi_{\nu} u_{v}\left(\varrho\left(2^{\nu}\right) \mid\right) d \mu=\sum_{\nu \in F} \int_{e_{\nu}} \varphi_{0}\left(|a| / \varrho\left(2^{\nu}\right)\right) d \mu\right. \\
& \leqslant C \sum_{\nu \in F^{\prime}} \int_{e_{\nu}} \varphi_{0}(|a| / \varrho(h(|a|))) d \mu=C \int_{M} \varphi(|a|) d \mu \leqslant C .
\end{aligned}
$$

Using the fact that $\varphi_{0}$ has positive lower type, we see that thus

$$
\left\|\sum_{\nu \in F} \xi_{\nu} u_{\nu} / \varrho\left(2^{\nu}\right)\right\|_{L^{\varphi_{0}}} \leqslant C
$$

In a similar way, we estimate $\left\|\sum_{\nu \in F} \xi_{\nu} 2^{v} u_{\nu} / \varrho\left(2^{\nu}\right)\right\|_{L^{\varphi_{1}}}$. This is much simpler since we do not need finite upper type (nor positive lower type). Indeed, we obtain the bound 1 . We conclude thus that $a \epsilon\left\langle\overrightarrow{L^{\varphi}}, \varrho\right\rangle$ with $\|a\|_{\left\langle L^{p}, \varrho\right\rangle} \leqslant$

Next we prove a result in the opposite sense.

THEOREM 7.2. Assume now - beyond what was assumed in Theorem 7.1 - that $\varphi_{1}$ too has finite upper type, and moreover, that $\varrho \epsilon \mathfrak{P}^{+-}(1)$. Then holds $\left\langle\overrightarrow{L^{\varphi}}, \varrho\right\rangle \subset L^{\varphi}$, continuous embedding.

Proof. Let $a \in\left\langle\overrightarrow{L^{\varphi}}, \varrho\right\rangle$. Again we may assume that $\|a\|_{\left\langle L^{\vec{\varphi}}, \varrho\right\rangle}<1$. Let $u=\left\{u_{\boldsymbol{v}}\right\}_{\boldsymbol{v} \in \boldsymbol{Z}}$ be a sequence satisfying (6.1) and (6.2) (with $\stackrel{\left\langle L^{\varphi}, Q\right\rangle}{C}=1$ ). We consider only sequences $\xi$ with $\xi_{\nu}= \pm 1$. Using Fubini's theorem, we get

$$
\int_{M} E\left(\varphi_{i}\left(\left|\sum_{\nu \in F} \pm 2^{i v} u_{v} / \varrho\left(2^{v}\right)\right|\right)\right) d \mu \leqslant 1 \quad(i=0,1) .
$$

By our Khintchine's inequality (Proposition 3.4) this gives

$$
\int_{M} \varphi_{i}\left(\left(\sum_{\nu \in F}\left(2^{i v} u_{v} / \varrho\left(2^{\nu}\right)\right)^{2}\right)^{1 / 2}\right) d \mu \leqslant C \quad(i=0,1) .
$$

Again our Oarlson's inequality (Corollary 3.1) in conjunction with Proposition 5.1, yields

$$
\int_{M} \varphi\left(\left|\sum_{\nu \in F} u_{\nu}\right|\right) d \mu \leqslant C
$$

or, after passing to the limit,

$$
\int_{M} \varphi(|a|) d \mu \leqslant C
$$

Now, in view of our assumptions, $\varphi$ has positive lower type, too. Therefore, we conclude that $a \in L^{\varphi}$ and $\|a\|_{L^{\varphi}} \leqslant C$.
Combining Theorems 7.1 and 7.2 with Remark 5.1 and using Proposition 6.1, we may summarize our findings as follows:

THEOREM 7.3. Let $\varphi_{0}$ and $\varphi_{1}$ be continuous increasing functions on $\boldsymbol{R}_{+}$ with $\varphi_{i}\left(\boldsymbol{R}_{+}^{\times}\right)=\boldsymbol{R}_{+}^{\times}(i=0,1)$, both of positive lower type and of finite upper type. Define $\varphi$ by (0.1) with $\varrho$ pseudoconcave in $\mathfrak{P}^{+-}(1)$. Then

$$
L^{\varphi}=\left\langle\overrightarrow{L^{\varphi}}, \varrho\right\rangle=L^{\varphi_{0}} \varrho\left(L^{\varphi_{1}} / L^{\varphi_{0}}\right)
$$

holds with equivalence of norms. In particular, $L^{\varphi}$ is an interpolation space with respect to $\overrightarrow{L^{\phi}}$, of type $s_{\mathrm{e}}$.

8. Some concrete illustrations. Recall the notation (see Example 2.4) $\varrho_{\theta \alpha \beta}(x)=x^{\theta}(\log (e+x))^{\alpha}(\log (e+1 / x))^{\beta}, \quad 0<\theta<1, \quad \alpha, \beta$ arbitrary real and, in particular (see Example 2.2),

$$
\varrho_{\theta}(x)=x^{\theta}, \quad 0<\theta<1 .
$$

Let us now also put

$$
\varphi_{p s t}(x)=x^{p}(\log (A+x))^{s}(\log (B+1 / x))^{t}, \quad p>0, s, t \text { arbitrary real, }
$$

where $A=A_{p s t}$ and $B=B_{p s t}$ are chosen so that $\varphi_{p s t}$ will be increasing, writing $\varphi_{p}=\varphi_{p 00}$. Set

$$
L^{p s t}=L^{\varphi_{p s t}}, \quad L^{p}=L^{\varphi_{p}} .
$$

We can then announce

Proposition 8.1. We have $\left\langle L^{p_{0} 8_{0} t_{0}}, L^{p_{1} s_{1} t_{1}}, \varrho_{\theta}\right\rangle=L^{\text {pst }}$ with $p, s, t d e-$ termined by

(8.1) $\quad 1 / p=(1-\theta) / p_{0}+\theta / p_{1}, \quad s / p=s_{0}(1-\theta) / p_{0}+s_{1} \theta / p_{1}$,

$$
t / p=t_{0}(1-\theta) / p_{0}+t_{1} \theta / p_{1} .
$$

Proof. It suffices to show that, if $\varphi$ is defined by (0.1) with $\varphi_{i}=\varphi_{p_{i} s_{i} t_{i}}$ $(i=0,1), \varrho=\varrho_{\theta}$, then $\varphi$ and $\varphi_{p s t}$ are equivalent. This follows essentially from the remark that, if $f(x)=x(\log x)^{r}$ in $[a, \infty), a>1$, then $f^{-1}(x)$ $\approx x(\log x)^{-r}$. We omit the details of the elementary argument.

Coroliary 8.1. Assume that the measure $\mu$ is finite. Then

$$
\left\langle L^{p_{0}}(\stackrel{+}{\log L} L)^{s_{0}}, L^{p_{1}} \stackrel{+}{+}(\log L)^{s_{1}}, \varrho_{\theta}\right\rangle=L^{p}(\stackrel{+}{\log L})^{8}
$$

holds with $p, s$ related by (8.1).

Remark 8.1. If $p_{0}=p_{1}=1, s_{0}=0, s_{1}=1$, compare Bennett [2], pp. 215, 224-225.

Proposition 8.2. Let $p_{0} \neq p_{1}$. We have $\left\langle L^{p_{0}}, L^{p_{1}}, \varrho_{\theta \alpha \beta}\right\rangle=I^{p s t}$ with $1 / p=(1-\theta) / p_{0}+\theta / p_{1}, s=-\beta p, t=-\alpha p$ (if $\left.p_{0}<p_{1}\right)$ or $s=-\alpha p$, $t=-\beta p\left(\right.$ if $\left.p_{0}>p_{1}\right)$. 
Proof. Put $\delta=1 / p_{1}-1 / p_{0}$. Using (0.1), we get

$$
\varphi^{-1}=x^{1 / p}\left(\log \left(e+x^{\delta}\right)\right)^{\alpha}\left(\log \left(e+x^{-\delta}\right)\right)^{\beta} .
$$

The result follows now in a similar way as in the proof of Proposition 8.1.

9. A limiting case. The purpose of this section is to prove the following generalization of Theorem 7.3.

THEOREM 9.1. Let $\varphi_{0}$ be a continuous increasing function on $\boldsymbol{R}_{+}$with $p_{0}\left(\boldsymbol{R}_{+}\right)=\boldsymbol{R}_{+}$and of positive lower type and finite upper type. Define $p$ by

$$
\varphi^{-1}=\varphi_{0}^{-1} \varrho\left(1 / \varphi_{0}^{-1}\right)\left(=\varrho^{*}\left(\varphi_{0}^{-1}\right)\right)
$$

with $\varrho$ pseudoconcave in $\mathfrak{B}^{+}(1)$. We also assume that $\varrho^{*}\left(\boldsymbol{R}_{+}^{\times}\right)=\boldsymbol{R}_{+}^{\times}$. (This is essentially a technical assumption; cf. Example 9.1 below.) Then

$$
L^{\varphi}=\left\langle L^{\varphi_{0}}, L^{\infty}, \varrho\right\rangle=L^{\varphi_{0}} \varrho\left(L^{\infty} \mid L^{\varphi_{0}}\right) .
$$

Proof. The idea of the proof is that formally $L^{\infty}=L^{\varphi_{1}}$, where

$$
\varphi_{1}(x)=\left\{\begin{array}{ccc}
0 & \text { if } & x \leqslant 1 \\
\infty & \text { if } & x>1
\end{array} .\right.
$$

Thus $L^{\infty}$ is a limiting case of Orlicz space. Having acquired this insight, it is not difficult to carry over the argument of the proof of Theorem 7.1 and 7.2 to the present case. (Note that the $\Delta_{2}$-condition was needed only for $\varphi_{0}$.) First we determine for each $z$ a unique $t=h(z)$ by the formula $t z=\varrho(t)$. Now using (9.1) there easily follows, corresponding to (7.1),

$$
\varphi(z)=\varphi_{0}(z / \varrho(t)) .
$$

Indeed, we get from (9.1)

$$
\varphi^{-1}\left(\varphi_{0}(z / \varrho(t))\right)=\varphi^{-1}\left(\varphi_{0}\left(t^{-1}\right)\right)=t^{-1} \varrho(t)=z
$$

which gives (9.2). Next, given $a \epsilon L^{\varphi}$ we define $e_{\nu}$ and $u_{\nu}$ as in Section 7 . The estimate (7.3) goes through unaltered. Thus we have

$$
\left\|\sum_{v \in F} \xi_{p} u_{p} / \varrho\left(2^{v}\right)\right\|_{L^{\varphi_{0}}} \leqslant C
$$

and it is trivial that

$$
\begin{aligned}
\left\|\sum_{\nu \in F^{\prime}} \xi_{\nu} 2^{v} u_{\nu} / \varrho\left(2^{\nu}\right)\right\|_{L^{\infty}} & \leqslant \max _{\nu \in F^{\prime}} \sup _{e_{\nu}} 2^{v}|a| \mid \varrho\left(2^{\nu}\right) \\
& \leqslant \max _{\nu \in F^{v}} \sup _{e_{\nu}} h(|a|)|a| / \varrho(h(|a|))=1 .
\end{aligned}
$$

Thus $a \epsilon\left\langle L^{\varphi_{0}}, L^{\infty}, \varrho\right\rangle$. This proves one inelusion $L^{\varphi} \subset\left\langle L^{\varphi_{0}}, L^{\infty}, \varrho\right\rangle$. There remains the converse.
Let $a \epsilon\left\langle L^{\varphi_{0}}, L^{\infty}, \varrho\right\rangle$ and pick up $u$ satisfying (6.1) and (6.2). As in the proof of Theorem 7.2, Khintchine's inequality gives

$$
\int_{M} \varphi_{0}\left(\left(\sum_{\nu \in F}\left(u_{v} / \varrho\left(2^{\nu}\right)\right)^{2}\right)^{1 / 2}\right) d \mu \leqslant C .
$$

On the other hand, we have for all choices of signs

$$
\left|\sum_{v \in F^{*}} \pm 2^{y} u_{v} / \varrho\left(2^{y}\right)\right| \leqslant 1 \mu \text {-a.e. }
$$

This implies

$$
\sum_{\nu \in F} 2^{\nu}\left|u_{v}\right| / \varrho\left(2^{\nu}\right) \leqslant 1 \quad \mu \text {-a.e. }
$$

Using directly (9.1), we now see that

$$
\int_{M} \varphi\left(\left(\sum_{\nu \in F}\left(u_{v} / \varrho\left(2^{v}\right)\right)^{2}\right)^{1 / 2} \varrho\left(\sum_{v \in F} 2^{v}\left|u_{v}\right| / \varrho\left(2^{p}\right) /\left(\sum_{v \in F^{\prime}}\left(u_{v} / \varrho\left(2^{v}\right)\right)^{2}\right)^{1 / 2}\right)\right) d \mu \leqslant C .
$$

Using thereafter Carlson's inequality in the form indicated in Remark 3.1, we find

$$
\int_{M} \varphi(|a|) d \mu \leqslant C
$$

and $a \epsilon L^{\varphi}$. This proves $\left\langle L^{\varphi_{0}}, L^{\infty}, \varrho\right\rangle \subset L^{\varphi}$.

EXAMPLE 9.1. (Application to Hausdorff-Young estimates). We consider the Fourier transform $\mathscr{F}$ on a compact Abelian group $G$ with (discrete) dual $\hat{G}$. (The classical case is: $G=\boldsymbol{R} / \boldsymbol{Z}, \hat{G}=\boldsymbol{Z}$.) Then

$$
\begin{aligned}
& \mathscr{F}: L^{2}(G) \rightarrow L^{2}(\hat{G}) \text { (Plancherel), } \\
& \mathscr{F}: L^{1}(G) \rightarrow L^{\infty}(\hat{G}) \text { ("Riemann-Lebesgue"). }
\end{aligned}
$$

We want to consider $\mathscr{F}$ on $L \log ^{+} L(G)$. To this end, it is natural to try to interpolate with $\varrho(x)=x / \log (e+x)$. Clearly; $\varrho \in \mathfrak{P}^{+}$. But $\varrho \notin \mathfrak{P}^{-}$and also $\varrho^{*}\left(\boldsymbol{R}_{+}^{\times}\right) \neq \boldsymbol{R}_{+}^{\times}$. Thus neither the full result of Section 7 (Theorem 7.3 ) nor the result of the present section (Theorem 9.1) are directly applicable. The first obstacle is not very serious since at least Theorem 7.1 applies. We get (cf. Proposition 8.2) the inclusion

$$
L \stackrel{+}{+} L \subset\left\langle L^{2}, L^{1}, \varrho\right\rangle \text {. }
$$

Regarding the second obstacle we can either modify the definition of $\varrho$ near 0 or use directly the proof of Theorem 9.1 properly modified. Anyway, we end up with the following result:

$$
f \in L+\stackrel{+}{\log L} L(G) \Rightarrow \sum_{\chi \in \hat{G}} e^{-C|\hat{f}(x)|^{-1}}<\infty \quad(\hat{f}=\mathscr{F} f) .
$$


A similar result holds, of course with $L\left(\log _{-}^{+} L\right)^{a}, a>0$, too (cf. Zygmund [18], Vol. II, p. 158).

10. A necessary condition. In this section we specialize the measure space to be $\boldsymbol{R}_{+}$equipped with the usual Lebesgue measure $d x$, and we take $\varphi_{i}=\varphi_{p_{i}}(i=0,1)$. (We write, of course, $L^{p}=L^{\varphi_{p}}$.) $\varphi$ is still expressed in terms of $\varphi_{0}$ and $\varphi_{1}$ with the aid of (0.1). We can now prove the following necessary condition for $\varrho$ (cf. Peetre [12], pp. 35-36).

Proposition 10.1. Assume that $L^{\varphi}$ is an interpolation space with respect to $\vec{L}^{p}=\left\{I^{p_{0}}, I^{p_{1}}\right\}$ (with $\vec{p}=\left(p_{0}, p_{1}\right)$ ), in the sense that $T: \overrightarrow{L^{p}} \rightarrow \overrightarrow{L^{p}}$ implies $T: L^{\varphi} \rightarrow L^{\varphi}$ and we have the inequality

$$
\|T\|_{L^{\varphi}, L^{\varphi}} \leqslant C \max \left(\|T\|_{L^{p_{0}, L^{p_{0}}}},\|T\|_{L^{p_{1}, L^{p_{1}}}}\right) .
$$

Then $\varrho$ must be pseudoconcave.

Proof. We choose $T a(x)=a(x / t), t>0$. It is plain that

Take now

$$
\|T\|_{L^{p_{i}, L}} p_{i}=t^{1 / p_{i}} \quad(i=0,1)
$$

$$
a(x)= \begin{cases}1 & \text { if } \quad 0<x<u \\ 0 & \text { otherwise }\end{cases}
$$

We readily see that

$$
\|a\|_{L^{\varphi}}=1 / \varphi^{-1}(1 / u), \quad\|T a\|_{L^{\varphi}}=1 / \varphi^{-1}(1 / t u) .
$$

Using (10.2) and (10.3) with (10.1), we subsequently get

$$
1 / \varphi^{-1}(1 / t u) \leqslant C \max \left(t^{1 / p_{0}}, t^{1 / p_{1}}\right) 1 / \varphi^{-1}(1 / u)
$$

or using (0.1)

$$
\varrho\left(u^{-\delta}\right) \leqslant C \max \left(1, t^{\delta}\right) \varrho\left((t u)^{-\delta}\right) \quad\left(\delta=1 / p_{1}-1 / p_{0}\right) .
$$

Stetting

$$
\lambda=t \delta, \quad x=(t u)^{-\delta},
$$

his gives $(2.1)$ and $\varrho \in \mathfrak{P}$.

Remark 10.1. If instead of (10.1) we require the stronger inequality

$$
\|T\|_{L^{p}, L^{p}} \leqslant O\|T\|_{L^{p_{0}, L^{p_{0}}}} f\left(\|T\|_{L^{p_{1}, L^{p_{1}}}} /\|T\|_{L^{p_{0}, L^{p_{0}}}}\right),
$$

the same argument shows that $f$ must satisfy

$$
\varrho(\lambda x) \leqslant O f(\lambda) \varrho(x),
$$

i.e. with $s_{Q}$ defined by (1.4) we have

$$
s_{e}(\lambda) \leqslant C f(\lambda) .
$$

\section{APPENDIX}

A. Interpolation of Orlicz classes. Below we reproduce, for the reader's convenience, the material of Peetre [12], pp. 36-37, in a slightly updated form, though (cf. Peetre-Sparr [16]).

Let $\varphi, \varphi_{0}$ and $\varphi_{1}$, as in Section 7 , be continuous increasing non-negative functions on $\boldsymbol{R}_{+}$. But assume now that they all have finite upper type (so that $O^{\varphi}, O^{\varphi_{0}}$ and $O^{\varphi_{1}}$ are F-spaces; we equip them with the quasi-F-norms $\|a\|_{O^{q}},\|a\|_{O^{\varphi_{0}}}$ and $\|a\|_{O^{\varphi_{1}}}$, respectively, defined in accordance with (5.1)) and that $\varphi$ is expressed in terms of $\varphi_{0}$ and $\varphi_{1}$ with the aid of $(0.3)$ with $\sigma$ pseudoconcave. We consider the "Orlicz (class) couple" $\vec{O}^{\vec{\varphi}}=\left\{O^{\varphi_{0}}, O^{\varphi_{1}}\right\}$.

THEOREM A.1. Consider any bounded linear mapping $T: \vec{O}^{\vec{\varphi}} \rightarrow O^{\vec{\varphi}}($ in the sinse that $T_{i}=T \mid O^{\varphi_{i}}: O^{\varphi_{i}} \rightarrow O^{\varphi_{i}}$ and we have $\left.\|T a\|_{O^{\varphi_{i}}} \leqslant C_{i}\|a\|_{O^{\varphi_{i}}}(i=0,1)\right)$. Then we have $T: O^{\varphi} \rightarrow O^{\varphi}$ and $\|T a\|_{O^{\varphi}} \leqslant C\|a\|_{O^{\varphi}}$.

Proof (outline). We set

$$
L(t, a)=\inf _{a=a_{0}+a_{1}}\left(\left\|a_{0}\right\|_{o^{\varphi_{0}}}+t\left\|a_{1}\right\|_{o^{\varphi_{1}}}\right) .
$$

Then our hypothesis on $T$ implies

$$
L(t, T a) \leqslant C L(t, a) .
$$

It is also easy to see that

$$
L(t, a) \approx \int_{M} \min \left(\varphi_{0}(|a|), t \varphi_{1}(|a|)\right) d \mu .
$$

It is no restriction to assume that $\sigma$ is concave (Proposition 2.1). For notational convenience mostly, let us also assume that

$$
\sigma(x)=o(\max (1, x)), \quad x \rightarrow 0 \text { or } \infty .
$$

Then we have the representation (cf. Peetre [14], p. 169)

$$
\sigma(x)=\int_{0}^{\infty} \min (1, x t) d w(t)
$$

where $d w$ is a positive measure on $\boldsymbol{R}_{+}^{\times}$. In view of (A.3), we now see, recalling also $(0.3)$, that

$$
\int_{0}^{\infty} L(t, a) d w(t) \approx\|a\|_{O^{\varphi}}
$$

and similarly for $T a$. In view of (A.2), this gives the desired result.

B. Weak type interpolation. We intend to give a generalization of Theorem (4.22) and Theorem (4.34) in Zygmund [18], Vol. II, p. 116 and p. 118, respectively. (Cf. the corresponding discussion in Peetre [12], pp. 31-33.) 
First we introduce the weak Orlicz class $O_{\text {weals }}^{p}$ by the condition

$$
a \in O_{\mathrm{weak}}^{\varphi} \Leftrightarrow \int_{\{|a| \geqslant x\}} d \mu \leqslant C / \varphi(x) .
$$

As in Section A, we always assume that $\varphi$ is continuous increasing nonnegative of finite upper type. Then $O_{\text {weak }}^{p}$ (as well as $O^{p}$ ) is an F-space, a quasi-F-norm being provided by

$$
\|a\|_{O_{\text {weak }}^{\varphi}}=\sup _{x>0} \dot{\varphi}(x) \int_{\{|a| \geqslant x\}} d \mu
$$

and we have $O^{\varphi} \subset O_{\text {wear }}^{\varphi}$, continuous embedding.

Let now $\varphi, \varphi_{\mathbf{0}}$ and $\varphi_{1}$ have the same significance as in Section $\mathbf{A}$. In particular, we thus assume (0.3).

THEOREM B.1. Assume further that $\varphi$ is absolutely continuous with

$$
\int_{0}^{\infty} \varphi^{\prime}(t) \min \left(\varphi_{0}(x) / \varphi_{0}(t), \varphi_{1}(x) / \varphi_{1}(t)\right) d t \leqslant C \varphi(x)
$$

and that $\sigma \epsilon \mathfrak{P}^{+-}$. Consider any bounded linear mapping $T: \vec{O}^{\vec{p}} \rightarrow \vec{O}_{\mathrm{weak}}^{\vec{\phi}}$ (in a similar sense as in Theorem A.1). Then we have $T: O^{\varphi} \rightarrow O^{\varphi}$ and $\|T a\|_{O^{\varphi}}$ $\leqslant C\|a\|_{O^{\varphi}}$.

Proof. Put $\omega(x, a)=\mu(\{|a| \geqslant x\})$. Then we have, as is well-known,

$$
\omega\left(x, a_{0}+a_{1}\right) \leqslant \omega\left(x / 2, a_{0}\right)+\omega\left(x / 2, a_{1}\right)
$$

and (since $\varphi$ is absolutely continuous)

$$
\text { (B.3) }\|a\|_{O^{p}}=\int_{M} \varphi(|a|) d \mu=-\int_{0}^{\infty} \varphi(x) d \omega(x, a)=\int_{0}^{\infty} \varphi^{\prime}(x) \omega(x, a) d x \text {. }
$$

Consider now any decomposition $a=a_{0}+a_{1}$ with $a_{i} \epsilon O_{\varphi_{i}}(i=0,1)$. Then from (B.2) applied with $T a_{i}$ and $\varphi_{i}(i=0,1)$ using the weak type estimate

follows

$$
\omega\left(x, T a_{i}\right) \leqslant \sigma_{i}\left\|a_{i}\right\|_{O^{p_{i}}} / p_{i}(x) \quad(i=0,1),
$$

$$
\omega(x, T a) \leqslant O\left(\left\|a_{0}\right\|_{O^{\varphi_{0}}} / \varphi_{0}(x)+\left\|a_{1}\right\|_{O^{\varphi_{1}}} / \varphi_{1}(x)\right) .
$$

In view of (A.1), this yields

$$
\omega(x, T a) \leqslant O 1 / \varphi_{0}(x) L\left(\varphi_{1}(x) / \varphi_{0}(x), a\right) .
$$

Next we apply (B.3), with $T a$, and (A.3). The result reads:

$$
\text { (B.5) }\|\boldsymbol{T} a\|_{O^{\varphi}} \leqslant C \int_{M} \int_{0}^{\infty} \varphi^{\prime}(x) \min \left(\varphi_{0}(|a|) / \varphi_{0}(x), \varphi_{1}(|a|) / \varphi_{1}(x)\right) d x d \mu .
$$

If we finally invoke (B.1), the proof is complete.
Now we discuss condition (B.1). Let us put $\gamma=\varphi_{1} / \varphi_{0}$. We assume that $\gamma$ is an increasing (absolutely continuous) function with $\gamma\left(\boldsymbol{R}_{+}^{\times}\right)=\boldsymbol{R}_{+}^{\times}$ and $\sigma \in \mathfrak{P}^{+-}(1)$. Then (B.1) can be written as

$$
\int_{0}^{\infty}\left(\varphi_{0}(t) \sigma(\gamma(t))\right)^{\prime} / \varphi_{0}(t) \min (1, \gamma(x) / \gamma(t)) d t \leqslant C \sigma(\gamma(x)) \text {. }
$$

Since

$$
\left(\varphi_{0} \sigma(\gamma)\right)^{\prime} / \varphi_{0}=\sigma^{\prime}(\gamma) \gamma^{\prime}+\varphi_{0}^{\prime} / \varphi_{0} \sigma(\gamma),
$$

the integral breaks up into two. The first one becomes, after a change of variables, viz. $\gamma(t)=\tau, \gamma(x)=\xi$, simply

$$
\int_{0}^{\infty} \sigma^{\prime}(\tau) \min (1, \xi / \tau) d \tau
$$

which in view of Remark 1.1 can be estimated in terms of

$$
\int_{0}^{\infty} \sigma(\tau) \min (1, \xi / \tau) d \tau / \tau
$$

which again can be estimated by $C \sigma(\xi)$. There remains the second integral, viz

$$
\int_{0}^{\infty} \varphi_{0}^{\prime}(t) / \varphi_{0}(t) \sigma(\gamma(t)) \min (1, \gamma(x) / \gamma(t)) d t .
$$

To treat it we need some additional hypothesis. One way out would be simply to postulate

$$
\text { (B.7) } \quad \varphi_{0}^{\prime} / \varphi_{0} \leqq C \gamma^{\prime} / \gamma \text {. }
$$

The same change of variables again leads to (B.6). It is easy to give further conditions which imply (B.7). For example, if we assume that $\varphi_{0} \epsilon \mathfrak{I}(0, p ; 1)$ for some $p$, then Remark 1.1 gives $x \varphi_{0}^{\prime}(x) \leqslant p \varphi_{0}(x)$ so (B.7) will be a consequence of $x \gamma^{\prime}(x) \geqslant q \gamma(x)(q>0)$ which again is fulfilled if $\gamma \in \mathfrak{I}(q, p ; 1)$. We summarize our findings in the following

CoRoulaRY B.1. Theorem B.1 is applicable when $\varphi_{0} \in \mathfrak{I}(0, p)$ for some $p<\infty$ and $\varphi_{1} / \varphi_{0} \in \mathfrak{I}(q, 1)$ for some $q>0$. (Analogous result with $\varphi_{0}$ and $p_{1}$ reversed.)

Proof. There remains just the application of Lemma 1.1.

Remark B.1. A quite different way out, applicable when $\varphi$ is in $\mathfrak{I}(0, p ; 1)$, is simply to postulate

(B.1 $\left.1^{\prime \prime}\right) \quad \int_{0}^{\infty} \varphi(t) \min \left(\varphi_{0}(x) / \varphi_{0}(t), \varphi_{1}(x) / \varphi_{1}(t)\right) d t / t \leqslant C \varphi(x)$.

Indeed, Remark 1.1 shows that (B.1.') implies (B.1). Condition (B.1') 
appears if we apply directly the method of [12]. There only the case $\varphi_{i}=\varphi_{p_{i}}(i=\theta, 1)$ is considered. In that case $\left(B .1^{\prime \prime}\right)$ and thus (B.1) are always fulfilled if $\sigma \epsilon \mathfrak{P}^{+-}$.

Remark B.2. If the measure $\mu$ is finite, we can somewhat relax the assumption of $\sigma$. Indeed, it suffices to assume that $\sigma \in \mathfrak{P}^{-}(1)$ and that

$$
\int_{1}^{x} \sigma(t) d t / t \leqslant O \sigma(x)
$$

With such a $\sigma$ we can just apply Theorem B.1 to $\bar{\sigma}$, defined by

$$
\bar{\sigma}(x)= \begin{cases}\sigma(x) & \text { if } x \geqslant 1 \\ x \sigma(1) & \text { if } x<1\end{cases}
$$

The result is only a slightly weaker, in the respect that we get the in . equality

We omit the details

$$
\|T a\|_{O^{\varphi}} \leqslant C_{1}\|a\|_{O^{\varphi}}+C_{2} .
$$

With Remark B.2 we have completely covered the case of Theorem (4.22) in [18], and we can safely turn to the case of Theorem (4.34). It is about the special case $\varphi_{0}=\varphi_{p_{0}}$, i.e. $L^{\varphi_{0}}=L^{1}$.

THEOREM B.2. Assume that $p$ is of the form

$$
\varphi(x)=x \int_{0}^{x} \chi(t) / t d t / t
$$

where $\chi$ is absolutely continuous increasing. Assume further that $\varphi(x) / x$ is increasing, that $\varphi(x)=o\left(\varphi_{1}(x)\right)$ as $x \rightarrow \infty$ and that (cf. $\left.\left(\mathrm{B1}^{\prime \prime}\right)\right)$

$$
\int_{x}^{\infty} \varphi(t) / \varphi_{1}(t) d t / t \leqslant O \varphi(x) / \varphi_{1}(x) .
$$

Finally, assume that $\varphi_{1}$ is differentiable with absolutely continuous derivative and satisfies

$$
\left(x / p_{1}(x)\right)^{\prime \prime} \leqslant O\left(1 /\left(x p_{1}(x)\right)\right)
$$

Consider any bounded linear mapping $T:\left\{L^{1}, O^{\phi_{1}}\right\} \rightarrow\left\{L_{\text {wealk }}^{1}, O_{\text {weak }}^{\varphi_{1}}\right\}$. Then we have $T: O^{\varphi} \rightarrow O^{x}$ and $\|T a\|_{O^{X}} \leqslant O\|a\|_{O^{\varphi}}$.

Proof. From the proof of Theorem B.1 (cf. in particular (B.5)), noting that $\chi^{\prime}(x)=x \varphi^{\prime \prime}(x)$, in view of (B.8), we see that we have to consider the integral

$$
I=\int_{0}^{\infty} \varphi^{\prime \prime}(t) \min \left(x, t \varphi_{1}(x) / \varphi_{1}(t)\right) d t
$$

Integrating by parts twice, we get

$$
\begin{aligned}
I & =-\int_{x}^{\infty} \varphi^{\prime}(t)\left(t \varphi_{1}(x) / \varphi_{1}(t)\right)^{\prime} d t \\
& \leqslant \int_{x}^{\infty} \varphi(t)\left(t \varphi_{1}(x) / \varphi_{1}(t)\right)^{\prime \prime} d t
\end{aligned}
$$

Using (B.9) and (B.1'"), we therefore obtain $I \leqslant C \varphi(x)$, which is all we need.

Remark B.3. As in the case of Theorem B.1 (see Remark B.2), the conditions can be somewhat relaxed in the case of a finite measure.

Remark B.4. It is needless to point out that all the results of this section, as always in the case of weak type interpolation, extend to the case of bounded quasi-sublinear operators. We can also allow different measures on both sides.

C. Application to Hausdorff-Young(-Paley) estimates. In this section we use the results of Section $B$ to prove two results which can be found in Bennett [3], p. 269. (Theorem C.2 can also be found in Zygmund [18], Vol. II, p. 158; the case $q=1$ is due to Hardy-Littlewood.)

We consider the Fourier transform $\mathscr{F}$ on $G=\boldsymbol{T}=\boldsymbol{R} / \boldsymbol{Z}$, the dual group $\hat{G}$ being as usual identified with $\boldsymbol{Z}$ (cf. Example 9.1). (Analogous results hold with $G=\boldsymbol{R}, \hat{G}=\boldsymbol{R}$.) We write $\hat{f}=\mathscr{F} f$ and denote by $\hat{f}^{*}$ the decreasing rearrangement of $\hat{f}$ on $N=1,2, \ldots$

THEOREM 0.1. If $\left.f \in L^{p} \stackrel{+}{(\log L}\right)^{q}, 1<p<2, q \geqslant 0$, then

$$
\sum_{n \in \boldsymbol{N}} n^{p-2}(\log n)^{q}\left(\hat{f}_{n}^{*}\right)^{p} \leqslant C \int_{\boldsymbol{T}}|f(x)|^{p}(\log |f(x)|)^{q} d x+C
$$

with $C$ independent of $f$.

Proof. Put $T f=\left\{n \hat{f}_{n}^{*}\right\}_{n \in \boldsymbol{N}}$. Then we have

$$
T: L^{1} \rightarrow l_{\text {weak }}^{1}, \quad T: L^{2} \rightarrow l^{2} \text {. }
$$

Here $L^{p}$ is with respect to Haar (Lebesgue) measure on $T$ and $l_{\text {(weak) }}^{p}$ with respect to the (discrete) measure on $N$ which to the point $n$ assigns mass $1 / n^{2}$. By Remark B.4, Theorem B.1 is still applicable. With $\varphi_{0}(x)=x$, $\varphi_{1}(x)=x^{2}, \sigma(x)=x^{p-1}(\log (e+x))^{q}$, we have $\varphi(x)=x^{p}(\log (e+x))^{q}$. The result reads :

(C.2) $\sum_{n \in N}\left(n \hat{f}_{n}^{*}\right)^{p}\left(\log \left(e+n \hat{f}_{n}^{*}\right)\right)^{q} \frac{1}{n^{2}} \leqslant C \int_{\boldsymbol{T}}|f(x)|^{p}(\log (e+|f(x)|))^{q} d x$.

It is easy to see that (C.2) entails (C.1) - consider separately the cases $n \hat{f}_{n}^{*}<\sqrt{n}$ and $n \hat{f}_{n}^{*} \geqslant \sqrt{n}-$ but we omit the details. 
THEOREM C.2. If $f \in L \stackrel{+}{\stackrel{+}{\log } L)^{q}}, q>0$, then

(C.3) $\quad \sum_{n \geqslant 2} n^{-1}(\log n)^{a-1} \hat{f}_{n}^{*} \leqslant C \int_{\boldsymbol{T}}|f(x)|\left(\log ^{+}|f(x)|\right)^{q} d x+C$.

Proof. We apply Theorem B.2 in the situation when

$$
\chi(x)=\left(x^{2} /(e+x)\right)(\log (e+x))^{q-1}
$$

so that (B.8) gives

We obtain

$$
\varphi(x)=q^{-1} x\left((\log (e+x))^{q}-1\right) .
$$

(C.4) $\sum_{n \in \mathbf{N}}\left(\left(\hat{f}_{n}^{*}\right)^{2} /\left(e+n \hat{f}_{n}^{*}\right)\right)\left(\log \left(e+n f_{n}^{*}\right)\right)^{a-1}$

$$
\leqslant C \int_{T}|f(x)|\left(\log (e+|f(x)|)^{q}-1\right) d x+C .
$$

Again we omit the somewhat tedious verification that (O.3) follows from (C.4).

\section{References}

[1] E. F. Beckenbach and R. Bellman, Inequalities, Exgebnisse der Mathematik N.F. 30, Springer-Verlag, Berlin, Heidelberg, New York 1965.

[2] C. Bennett, Intermediate spaces and the class $L \log X$, Ark. Mat. 11 (1973), pp. 215-228.

[3] - Banach function spaces and interpolation methods III. Hausdorff-Young estimates, J. Approximation Theory 13 (1975), pp. 267-275.

[4] A. P. Calderón, Intermediate spaces and interpolation, the complex method, Studia Math. 24 (1964), pp. 113-190.

[5] E. Gagliardo, Oaratterizzazione construttiva di tutti gli spazi di interpolazione tra spazi di Banach, Syposia Mathematica 2 (1968), pp. 95-106.

[6] J. Gustavsson, Metrization of quasi-metric spaces, Math. Scand. 35 (1974), pp. 56-60.

[7] E. Hille and R. S. Phillips, Functional analysis and semi-groups, A.M.S., Providence 1957

[8] G. Köthe, Topologische lineare Räume, Grundlehren der Matematischen Wissensohaften 107, Springer-Verlag, Berlin, Göttingen, Heidelberg 1960.

[9] M. A. Krasnoselskii and Ya. B. Rutitskii, Oonvex funotions and Orlioz spaces, Gos. Izdat. Fiz.-mat. Lit., Moscow 1958 (in Russian).

[10] W. T. Kraynek, Interpolation of sublinear operators in generalized Orlicz and Hardy-Orlicz spaces, Studia Math. 43 (1972), pp. 93-123.

[11] J. Musielak and W. Orlicz, On modular spaces, Studia Math. 18 (1959), pp. 49-65.

[12] J. Peetre, $A$ new approach in interpotation spaces, Studia Math. 34 (1970), pp. 23-42.

[13] - Sur l'utilisation des suites inconditionellement sommables dans la théorie des espaces d'interpolation, Rend. Sem. Mat. Padova 46 (1971), pp. 173-190.
[14] - On interpolation functions, Acta Szeged 27 (1966), pp. 167-171.

[15] - On interpolation functions II, ibid. 29 (1968), pp. 91-92

[16] J. Peetre and G. Sparr, Interpolation of nörmed Abelian groups, Ann. Mat. Pura Appl. 92 (1972), pp. 217-262.

[17] M. M. R ao, Interpolation, ergodicity and martingales, J. Math. Mech. 16 (1966), pp. $543-567$. [18] A. Zygmund, Trigonometrical series, Cambridge University Press, Cambridge
1958.

DEPARTMENT OF MATHEMATICS, LUND INSTITUTE OF TECHNOLOGY UNIVERSITY OF LUND, LUND, SWEDEN 